\title{
A thermodynamically consistent kinetic framework for binary nucleation
}

\author{
Richard C. Flagan ${ }^{\text {a) }}$ \\ Division of Chemistry and Chemical Engineering, California Institute of Technology, \\ Pasadena, California 91125, USA
}

(Received 30 July 2007; accepted 26 September 2007; published online 5 December 2007)

\begin{abstract}
The traditional theory for binary homogeneous nucleation follows the classical derivation of the nucleation rate in the supposition of a hypothetical constrained-equilibrium distribution in the calculation of the cluster evaporation rate. This model enables calculation of the nucleation rate, but requires evaluation of the cluster distribution and cluster properties for an unstable equilibrium with supersaturated vapor. An alternate derivation of the classical homomolecular nucleation rate eliminated the need for this nonphysical approximation by calculating the evaporative flux at full thermodynamic equilibrium. The present paper develops that approach for binary nucleation; the framework is readily extended to ternary nucleation. In this analysis, the evaporative flux is evaluated by applying mass balance at full thermodynamic equilibrium of the system under study. This approach eliminates both the need for evaluating cluster properties in an unstable constrained-equilibrium state and ambiguity in the normalization constant required in the nucleation-rate expression. Moreover, it naturally spans the entire composition range between the two pure monomers. The cluster fluxes derived using this new model are similar in form to those of classical derivations, so previously developed methods for evaluation of the net nucleation rate can be applied directly to the new formulation. (C) 2007 American Institute of Physics.
\end{abstract}

[DOI: $10.1063 / 1.2800001]$

\section{INTRODUCTION}

Binary and ternary nucleation have received considerable attention in recent years due to the growing recognition of the significance of these new particle formation mechanisms to the atmospheric aerosol, and to a wide range of other problems in science and technology. Stratospheric aerosols that catalyze ozone depletion are thought to result from binary nucleation. ${ }^{1}$ Although it has long been presumed that tropospheric aerosol concentrations were sufficiently high to suppress new particle formation, recent studies have shown that multicomponent nucleation generates substantial concentrations of new, ultrafine particles in environments ranging from the boreal forest ${ }^{2,3}$ to polluted urban atmospheres. ${ }^{3,4}$

The theory of the rate of binary nucleation describes the passage of clusters over a saddle point in the free energy of cluster formation. Reiss ${ }^{5}$ first derived the theoretical rate of binary nucleation of liquid droplets from a mixture of vapors by assuming that the cluster flux over the saddle follows the direction of steepest descent on the free energy surface in the vicinity of the saddle point. Later investigators ${ }^{6-8}$ showed that the direction of passage deviates from that direction due to the kinetics of cluster growth, and developed concise and general equations for evaluating the total rate of formation of stable clusters. Numerous studies have attempted to improve theoretical predictions by focusing on the nature of the free energy surface. ${ }^{1,9-12}$ A common thread through these studies is the evaluation of a cluster distribution that is equilibrated with supersaturated vapor. To maintain this unstable equilib-

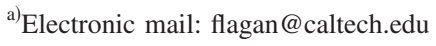

rium would require a constraint, sometimes portrayed as a Maxwell demon, to preclude the formation of clusters well beyond the critical cluster size. As in the classical homomolecular nucleation theory, the supersaturated equilibrium is introduced in the determination of the rate of monomer loss from growing clusters by using a kinetic theory estimate of the rate of monomer addition in the supersaturated state and applying the law of mass action to determine the reverse rate. Numerous investigators have reported on efforts to improve the estimation of this hypothetical constrained-equilibrium cluster distribution by imposing self-consistency conditions on the cluster surface free energy. ${ }^{13-17}$

In an alternate derivation of the homomolecular (singlecomponent) homogeneous nucleation rate, Katz and Wiedersich $^{18}$ (KW) showed that the hypothetical constrained-equilibrium cluster distribution is an unnecessary artifice. Instead of evaluating the evaporative flux at the supersaturated state, mass action was applied at full thermodynamic equilibrium to determine that flux. The resulting steady-state rate expression was the same as the classical rate, but the ambiguity involved in evaluating the constrained-equilibrium cluster distribution and in estimating cluster properties, such as surface free energy, at a supersaturated state was eliminated. The KW model has previously been applied to a number of homomolecular nucleation problems, including nucleation of single pure phases in condensed systems, ${ }^{19}$ and particle formation in systems undergoing chemical reactions. ${ }^{20}$

The present paper extends the KW model to binary and multicomponent nucleation. The paper begins with a brief reprise of the classical binary nucleation theory, derives the extended KW nucleation model, and then examines the equi- 
librium cluster distribution in order to enable comparison between the new kinetic model and the classical form. The new model resolves a long-standing question about the normalization constant that is required to evaluate the equilibrium (or constrained-equilibrium) cluster distribution, naturally reducing to the expected result in the unary (singlecomponent) cluster limit.

\section{TRADITIONAL BINARY NUCLEATION THEORY}

Binary nucleation can be described as the kinetics of a number of reversible monomer addition reactions. The net rate of addition of $A$ molecules to an $A_{i} B_{j}$ cluster is

$$
J_{i, j}^{A}=\beta_{A} s_{i, j} N_{i, j}-e_{i+1, j}^{A} N_{i+1, j},
$$

and the rate of addition of $B$ molecules is

$$
J_{i, j}^{B}=\beta_{B} s_{i, j} N_{i, j}-e_{i, j+1}^{B} N_{i, j+1},
$$

where $\beta_{\chi}$ denotes the flux of monomer $\chi$ to the clusters, $s_{i, j}$ is the surface area of an $\{i, j\}$ cluster, $N_{i, j}$ is the concentration of $\{i, j\}$ clusters, and $e_{i, j}^{\chi}$ is the frequency of evaporation of a molecule of species $\chi$ from an $\{i, j\}$ cluster. New particles form directly from the vapor phase when the rate of monomer addition to clusters of a critical size exceeds the rate of evaporation of clusters of that size. This occurs when the vapor phase is supersaturated with respect to an equilibrium condensed phase.

Calculation of that nucleation rate requires determination of the rates of addition of both monomers to the cluster, and the rates at which both monomers evaporate from the cluster. The monomer arrival rates can be calculated from the kinetic theory of gases. The evaporation rate is calculated by applying the principle of microscopic reversibility at a hypothetical, constrained-equilibrium state wherein the clusters are equilibrated with the supersaturated vapors. In this hypothetical state, the cluster flux must be zero to maintain the constrained equilibrium, i.e.,

$$
\begin{aligned}
& J_{i, j}^{A, c e}=\beta_{A} s_{i, j} N_{i, j}^{c e}-e_{i+1, j}^{A} N_{i+1, j}^{c e}=0, \\
& J_{i, j}^{B, c e}=\beta_{B} s_{i, j} N_{i, j}^{c e}-e_{i, j+1}^{B} N_{i, j+1}^{c e}=0,
\end{aligned}
$$

from which

$$
\begin{aligned}
& e_{i+1, j}^{A}=\beta_{A} s_{i, j} N_{i, j}^{c e} / N_{i+1, j}^{c e}, \\
& e_{i, j+1}^{B}=\beta_{B} s_{i, j} N_{i, j}^{c e} / N_{i, j+1}^{c e} .
\end{aligned}
$$

Substituting these evaporation coefficients into Eqs. (3) and (4) yields

$$
\begin{aligned}
& J_{i, j}^{A}=\beta_{A} s_{i, j} N_{i, j}^{c e}\left(\frac{N_{i, j}}{N_{i, j}^{c e}}-\frac{N_{i+1, j}}{N_{i+1, j}^{c e}}\right), \\
& J_{i, j}^{B}=\beta_{B} s_{i, j} N_{i, j}^{c e}\left(\frac{N_{i, j}}{N_{i, j}^{c e}}-\frac{N_{i, j+1}}{N_{i, j+1}^{c e}}\right) .
\end{aligned}
$$

For sufficiently large $i$ and $j, N_{i, j}$ and $N_{i, j}^{c e}$ can be replaced with continuous functions of $i$ and $j$, and the differences can be approximated by derivatives, leading to

$$
\begin{aligned}
& J^{A}(i, j) \approx-\beta_{A} s(i, j) n^{c e}(i, j) \frac{\partial}{\partial i}\left(\frac{n(i, j)}{n^{c e}(i, j)}\right), \\
& J^{B}(i, j) \approx-\beta_{B} s(i, j) n^{c e}(i, j) \frac{\partial}{\partial j}\left(\frac{n(i, j)}{n^{c e}(i, j)}\right) .
\end{aligned}
$$

The time rate of change of clusters of size $\{i, j\}$ is then

$$
\begin{aligned}
\frac{\partial n(i, j)}{\partial t}= & J_{i-1, j}^{A}-J_{i, j}^{A}+J_{i, j-1}^{B}-J_{i, j}^{B} \\
\approx & \frac{\partial}{\partial i}\left[\beta_{A} s(i, j) n^{c e}(i, j) \frac{\partial}{\partial i}\left(\frac{n(i, j)}{n^{c e}(i, j)}\right)\right] \\
& +\frac{\partial}{\partial j}\left[\beta_{B} s(i, j) n^{c e}(i, j) \frac{\partial}{\partial j}\left(\frac{n(i, j)}{n^{c e}(i, j)}\right)\right] .
\end{aligned}
$$

The rate of new particle formation can be evaluated by solving for the transient population of $\{i, j\}$ clusters using Eq. (11) numerically, ${ }^{10}$ or, more commonly, by solving for the steady-state nucleation rate by setting $\partial n(i, j) / \partial t=0$. Both approaches require evaluation of the constrained-equilibrium cluster distribution, which is usually cast in the form

$$
N_{i, j}^{c e}=n^{c e}(i, j)=N_{0} e^{-\omega_{i, j}},
$$

where the dimensionless reversible work of forming the $\{i, j\}$ cluster is generally taken to be

$\omega_{i, j}=\frac{4 \pi R_{P}^{2} \sigma_{i, j}}{k T}+i \ln \left(\gamma_{A}^{i, j} x_{\chi}^{i, j}\right)+j \ln \left(\gamma_{B}^{i, j} x_{B}^{i, j}\right)-i \ln S_{A}-j \ln S_{B}$

Here $R_{p}$ is the radius of the cluster, $\sigma_{i, j}$ is the surface tension of a cluster of size $\{i, j\}, x_{\chi}^{i, j}$ and $\gamma_{\chi}^{i, j}$ are the mole fraction and activity coefficient of species $\chi$ in a bulk solution with the composition of that in the core of a cluster consisting of $i$ molecules of $A$ and $j$ molecules of $B$, respectively, and $S_{\chi}$ $=p_{\chi} / p_{\chi}^{\text {sat }}$ is the saturation ratio for species $\chi$. In the special case that the composition is uniform throughout the cluster, i.e., one in which the binary system does not exhibit surface activity, and the surface tension is not a function of composition, the mole fraction in the cluster solution becomes $x_{A}^{i, j}$ $=i /(i+j)$, and the activity in the cluster solution is the same as that in a bulk solution of the same composition. On the other hand, if the binary system does exhibit surface active behavior, $A$ and $B$ will not partition equally between the core solution and the surface of the cluster. Laaksonen et al. ${ }^{21}$ provide a detailed discussion of approaches for treating this more complex problem.

Reiss ${ }^{5}$ suggested that the reference vapor concentration should be $N_{0}=N_{A}+N_{B}$, although this leads to incorrect results in the unary limit, ${ }^{10}$ i.e., for clusters containing either pure $A$ or pure $B$. Wilemski and Wyslouzil ${ }^{10}$ developed an $a d$ $h o c$ construction for the reference vapor concentration that overcomes this weakness. The physical interpretation of this scaling factor remains uncertain.

Following Stauffer ${ }^{6}$ and Trinkaus $^{7}$ the cluster balance equation, Eq. (11), can be expressed concisely as 


$$
\frac{d n(\mathbf{i})}{\partial t}=-\nabla \mathbf{J}=\nabla\left[\mathbf{R} n^{c e}(\mathbf{i}) \cdot \nabla\left(\frac{n(\mathbf{i})}{n^{c e}(\mathbf{i})}\right)\right],
$$

where $\mathbf{J}$ is the cluster current vector in composition space, $\mathbf{i}$, and the matrix $\mathbf{R}=\beta s(\mathbf{i})$ represents the monomer attachment rate to a cluster $\mathbf{i}$, which may include off diagonal elements if vapors associate, e.g., hydrates in sulfuric acid/water binary nucleation. The steady-state nucleation rate is found by expanding $\omega(\mathbf{i})$ about the $\mathbf{i}^{*}$ cluster corresponding to a saddle point in $\omega$ up to second order terms, i.e.,

$$
\omega \approx \omega^{*}+\frac{1}{2} \Delta \mathbf{i} \cdot \omega^{*} \cdot \Delta \mathbf{i} .
$$

The resulting steady-state nucleation rate is

$$
J=\frac{|\lambda| /(2 \pi)}{\sqrt{\operatorname{det}\left[\omega^{*} /(2 \pi)\right]}} N_{0} e^{-\omega^{*}},
$$

where $\lambda$ is the negative eigenvalue of $\mathbf{R} \cdot \mathbf{w}$. In this formulation, passage over the saddle proceeds in a direction determined by the kinetic parameters; Wilemski ${ }^{8}$ obtained an equivalent result in terms of the orientation angles of the saddle and the cluster fluxes.

\section{KINETIC THEORY OF BINARY NUCLEATION}

Katz and Wiedersich ${ }^{18}$ eliminated the hypothetical constrained equilibrium in homomolecular (single-component) nucleation by evaluating the evaporation rate at full thermodynamic equilibrium where, as earlier observed by McDonald, ${ }^{22}$ clusters are present at concentrations that generally decrease continuously with increasing cluster size. Following their approach for binary nucleation, we note that the equilibrium cluster fluxes are

$$
\begin{aligned}
& J_{i, j}^{A, e} \equiv 0=\beta_{A}^{e} s_{i, j} N_{i, j}^{e}-e_{i+1, j}^{A, e} N_{i+1, j}^{e}, \\
& J_{i, j}^{B, e} \equiv 0=\beta_{B}^{e} s_{i, j} N_{i, j}^{e}-e_{i, j+1}^{B, e} N_{i, j+1}^{e},
\end{aligned}
$$

where the cluster distribution, $N_{i, j}^{e}$, is evaluated at the relevant full thermodynamic equilibrium state. The equilibrium evaporation rate coefficients then become

$$
\begin{aligned}
& e_{i+1, j}^{A, e}=\beta_{A}^{e} s_{i, j} N_{i, j}^{e} / N_{i+1, j}^{e}, \\
& e_{i, j+1}^{B, e}=\beta_{B}^{e} s_{i, j} N_{i, j}^{e} / N_{i, j+1}^{e},
\end{aligned}
$$

where $\beta_{\chi}^{e}$ denotes the flux of monomer $\chi$ to the surface of the cluster at full thermodynamic equilibrium. Assuming that the evaporation flux depends on the state of the cluster, and not on that of the surrounding gas, i.e.,

$$
e_{i, j}^{\chi}=e_{i, j}^{\chi, e},
$$

the cluster growth rates can now be written as

$$
\begin{gathered}
J_{i, j}^{A}=\beta_{A} s_{i, j} N_{i, j}-\beta_{A}^{e} s_{i, j} N_{i, j}^{e} \frac{N_{i+1, j}}{N_{i+1, j}^{e}}, \\
J_{i, j}^{B}=\beta_{B} s_{i, j} N_{i, j}-\beta_{B}^{e} s_{i, j} N_{i, j}^{e} \frac{N_{i, j+1}}{N_{i, j+1}^{e}} .
\end{gathered}
$$

This may be rearranged to form

$$
\begin{gathered}
J_{i, j}^{A}=\beta_{A} s_{i, j} N_{i, j}^{e}\left[\frac{N_{i, j}}{N_{i, j}^{e}}-\frac{N_{i+1, j}}{N_{i+1, j}^{e}} \frac{\beta_{A}^{e}}{\beta_{A}}\right], \\
J_{i, j}^{B}=\beta_{B} s_{i, j} N_{i, j}^{e}\left[\frac{N_{i, j}}{N_{i, j}^{e}}-\frac{N_{i, j+1}}{N_{i, j+1}^{e}} \frac{\beta_{B}^{e}}{\beta_{B}}\right] .
\end{gathered}
$$

In order to recover the symmetry of condensation and evaporation terms that enables solution for the steady-state nucleation rate, KW (Ref. 18) multiplied and divided by the ratio of the actual and equilibrium rates of the monomer fluxes raised to the appropriate power. Following their approach, we find

$$
\begin{aligned}
& J_{i, j}^{A}=\beta_{A} s_{i, j} N_{i, j}^{e}\left(\frac{\beta_{A}}{\beta_{A}^{e}}\right)^{i}\left[\frac{N_{i, j}}{N_{i, j}^{e}}\left(\frac{\beta_{A}^{e}}{\beta_{A}}\right)^{i}-\frac{N_{i+1, j}}{N_{i+1, j}^{e}}\left(\frac{\beta_{A}^{e}}{\beta_{A}}\right)^{i+1}\right], \\
& J_{i, j}^{B}=\beta_{B} s_{i, j} N_{i, j}^{e}\left(\frac{\beta_{B}}{\beta_{B}^{e}}\right)^{j}\left[\frac{N_{i, j}}{N_{i, j}^{e}}\left(\frac{\beta_{B}^{e}}{\beta_{B}}\right)^{j}-\frac{N_{i, j+1}}{N_{i, j+1}^{e}}\left(\frac{\beta_{B}^{e}}{\beta_{B}}\right)^{j+1}\right] .
\end{aligned}
$$

While Eqs. (26) and (27) are recursive in terms of the individual species, they do not provide the full symmetry needed to recover the classical result for multicomponent nucleation. An additional multiplication and division brings the result we seek, which can be expressed concisely as

$$
\begin{aligned}
& J_{i, j}^{A}=\beta_{A} s_{i, j} F_{i, j}\left[\frac{N_{i, j}}{F_{i, j}}-\frac{N_{i+1, j}}{F_{i+1, j}}\right], \\
& J_{i, j}^{B}=\beta_{B} s_{i, j} F_{i, j}\left[\frac{N_{i, j}}{F_{i, j}}-\frac{N_{i, j+1}}{F_{i, j+1}}\right],
\end{aligned}
$$

where

$$
F_{i, j}=N_{i, j}^{e}\left(\frac{\beta_{A}}{\beta_{A}^{e}}\right)^{i}\left(\frac{\beta_{B}}{\beta_{B}^{e}}\right)^{j} .
$$

It is important to note that $\beta_{A}^{e}$ and $\beta_{B}^{e}$ represent the fluxes of $A$ and $B$ at the same equilibrium state used to evaluate $N_{i, j}^{e}$ rather than at the saturation partial pressures over pure liquid of that species.

For large $i$ and $j$, the properties of the clusters and the cluster distribution can be expressed as continuous functions, i.e.,

$$
\begin{aligned}
& J^{A}(i, j) \approx-\beta_{A} s(i, j) f(i, j) \frac{\partial}{\partial i}\left(\frac{n(i, j)}{f(i, j)}\right), \\
& J^{B}(i, j) \approx-\beta_{B} s(i, j) f(i, j) \frac{\partial}{\partial j}\left(\frac{n(i, j)}{f(i, j)}\right),
\end{aligned}
$$

as before. The time rate of change of $\{i, j\}$ clusters is then

$$
\begin{aligned}
\frac{\partial n(i, j)}{\partial t} \approx & \frac{\partial}{\partial i}\left[\beta_{A} s(i, j) f(i, j) \frac{\partial}{\partial i}\left(\frac{n(i, j)}{f(i, j)}\right)\right] \\
& +\frac{\partial}{\partial j}\left[\beta_{B} s(i, j) f(i, j) \frac{\partial}{\partial j}\left(\frac{n(i, j)}{f(i, j)}\right)\right],
\end{aligned}
$$

which is identical in form to Eq. (11), although $f(i, j)$ can no longer be interpreted as the constrained-equilibrium (or equilibrium) cluster distribution. 
Extension to ternary nucleation of species $A, B$, and $C$ is straightforward, yielding

$$
\begin{aligned}
& J_{i, j, k}^{A}=\beta_{A} s_{i, j, k} F_{i, j, k}\left[\frac{N_{i, j, k}}{F_{i, j, k}}-\frac{N_{i+1, j, k}}{F_{i+1, j, k}}\right], \\
& J_{i, j, k}^{B}=\beta_{B} s_{i, j, k} F_{i, j, k}\left[\frac{N_{i, j, k}}{F_{i, j, k}}-\frac{N_{i, j+1, k}}{F_{i, j+1, k}}\right], \\
& J_{i, j, k}^{C}=\beta_{C} s_{i, j, k} F_{i, j, k}\left[\frac{N_{i, j, k}}{F_{i, j, k}}-\frac{N_{i, j, k+1}}{F_{i, j, k+1}}\right],
\end{aligned}
$$

where

$$
F_{i, j, k}=N_{i, j, k}^{e}\left(\frac{\beta_{A}}{\beta_{A}^{e}}\right)^{i}\left(\frac{\beta_{B}}{\beta_{B}^{e}}\right)^{j}\left(\frac{\beta_{C}}{\beta_{C}^{e}}\right)^{k} .
$$

\section{EQUILIBRIUM CLUSTER DISTRIBUTION}

The equilibrium cluster distribution must be known to evaluate the nucleation rate. We are considering a system in which multiple vapors are present, initially in a supersaturated state. The classical theory of binary nucleation considers the distribution of clusters in a constrained equilibrium with the supersaturated vapor, whose composition is specified. The present analysis focuses on the state of full thermodynamic equilibrium. This is a well-defined state, although the concentrations of the vapor species will differ from the initial state. Because of differences in the volatility of the vapors, even the relative vapor concentrations will differ from the initial state once condensation begins. The equilibrium composition of the vapor phase is, however, readily determined. The discussion that follows will evaluate that equilibrium state, and show that use of the full thermodynamic equilibrium cluster distribution resolves the longstanding ambiguity in the cluster concentration normalization constant that has been the subject of extensive discussion in the nucleation literature. ${ }^{10}$

Any system containing the two vapors in equilibrium over a bulk solution can produce clusters with the entire range of possible compositions, but the equilibrium partial pressures will vary depending on the starting composition. At equilibrium between pure liquid and pure vapor, the partial pressure of vapor of species $\chi$ is $p_{\chi}=p_{\chi}^{\text {sat }}$ and $\mu_{\chi}^{0}$ $+k T \ln p_{\chi}^{\text {sat }} / p_{v}^{e}=\mu_{\chi}^{*}$. For equilibrium over a solution of $A$ and $B, \mu_{\chi}^{0}+k T \ln p_{\chi}^{e} / p_{v}^{e}=\mu_{\chi}^{*}+k T \ln \gamma_{\chi}^{e} x_{\chi}^{e}$, where $p_{\chi}^{e}$ is the equilibrium partial pressure of species $\chi$ over a solution with mole fraction $x_{\chi}, p_{\chi}^{\text {sat }}$ is the saturation vapor pressure, and $\gamma_{\chi}^{e}$ is the activity coefficient for species $\chi$ in that equilibrium solution. The reference pressure is arbitrary, here taken to be $p_{v}^{e}$, the total vapor pressure. From this, we may write the extended form of Raoult's law,

$$
p_{\chi}^{e}=\gamma_{\chi}^{e} x_{\chi}^{e} p_{\chi}^{\mathrm{sat}}
$$

To determine the partial pressures of each of the species in the system, it is necessary to determine the partitioning of all vapors between the vapor and liquid solution phases.
The superscript $e$ serves as a reminder that the activity coefficients and solution mole fractions for all species are evaluated at the state of full thermodynamic equilibrium.

Before deriving the equilibrium composition, it is useful to examine the cluster distribution in the equilibrium state. The cluster $A_{i} B_{j}$ can be viewed as the product of the "reaction"

$$
i A+j B \rightleftharpoons A_{i} B_{j} .
$$

At equilibrium, the Gibbs free energies for the cluster vapor and the monomeric vapor reactants are equal,

$$
\begin{aligned}
\mu_{i, j}= & \mu_{i, j}^{0}+k T \ln \frac{p_{i, j}^{e}}{p_{v}^{e}}=i \mu_{A}^{0}+i k T \ln \frac{p_{A}^{e}}{p_{v}^{e}}+j \mu_{B}^{0} \\
& +j k T \ln \frac{p_{B}^{e}}{p_{v}^{e}}
\end{aligned}
$$

where the $A_{i} B_{j}$ vapor is assumed to be an ideal gas with partial pressure $p_{i, j}^{e}$. The cluster can be modeled as a droplet of liquid with free energy corresponding to bulk liquid with composition $x_{A}^{i, j}$, but corrected for finite size and, if necessary, surface activity effects by introducing a term, $\Gamma_{i, j}$, i.e.,

$$
\mu_{i, j}^{0}=i \mu_{A}^{*}+i k T \ln \left(\gamma_{A}^{i, j} x_{A}^{i, j}\right)+j \mu_{B}^{*}+j k T \ln \left(\gamma_{B}^{i, j} x_{B}^{i, j}\right)+\Gamma_{i, j}
$$

Only if the binary system does not exhibit surface activity will the mole fractions correspond to the ratios of the total numbers of molecules in the cluster, i.e., only then will $x_{A}^{i, j}$ $=i /(i+j)$. In that case, $\Gamma_{i, j}=4 \pi R_{p}^{2} \sigma$ will then be a function of size, but will not depend on the cluster composition. On the other hand, if the binary system does exhibit surface activity, the mole fractions will be those in the core liquid of the $\{i, j\}$ cluster and the analysis becomes considerably more complex. The methods described by Laaksonen et $a .^{21}$ can then be applied to properly define the surface of tension and evaluate $\Gamma_{i, j}$.

Substituting Eq. (40) into Eq. (39) and rearranging yields the equilibrium cluster partial pressure,

$$
\frac{p_{i, j}^{e}}{p_{v}^{e}}=\left(\frac{p_{A}^{e}}{p_{v}^{e}}\right)^{i}\left(\frac{p_{B}^{e}}{p_{v}^{e}}\right)^{j} \exp \left(-i \ln \gamma_{A}^{j, j} x_{A}^{i, j}-j \ln \gamma_{B}^{j, j} x_{B}^{i, j}-\frac{\Gamma_{i, j}}{k T}\right) .
$$

The cluster number concentration is simply $N_{i, j}^{e}=p_{i, j}^{e} / k T$. If one further substitutes Eq. (38), the cluster vapor pressure can be expressed as products of the saturation vapor pressures and ratios of the activities in the equilibrium bulk solution to those in the cluster solution, i.e.,

$$
\begin{aligned}
\frac{p_{i, j}^{e}}{p_{v}^{e}}= & \left(\frac{p_{A}^{\mathrm{sat}} \gamma_{A}^{e} x_{A}^{e}}{p_{v}^{e}}\right)^{i}\left(\frac{p_{B}^{\mathrm{sat}} \gamma_{B}^{e} x_{B}^{e}}{p_{v}^{e}}\right)^{j} \\
& \times \exp \left(-i \ln \gamma_{A}^{i, j} x_{A}^{i, j}-j \ln \gamma_{B}^{i, j} x_{B}^{i, j}-\frac{\Gamma_{i, j}}{k T}\right) .
\end{aligned}
$$




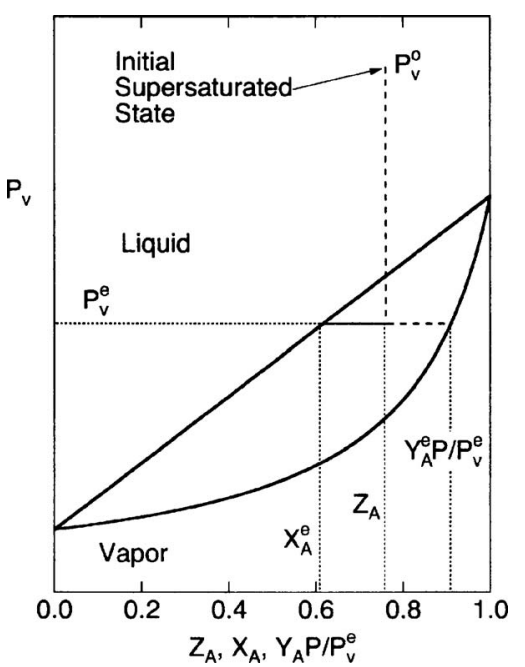

FIG. 1. Phase diagram for a two vapor system showing the equilibrium partitioning between liquid and vapor phases of a system that is initially supersaturated.

Equation (42) has another important property. In the limit where $j \rightarrow 0$,

$$
\begin{aligned}
\lim _{j \rightarrow 0} \frac{p_{i, j}^{e}}{p_{v}^{e}}=\frac{p_{i, 0}^{e}}{p_{v}^{e}} & =\left(\frac{p_{A}^{\text {sat }} \gamma_{A}^{e} x_{a}^{e}}{p_{v}^{e}}\right)^{i} \exp \left(-\frac{\Gamma_{i, 0}}{k T}\right) \\
& =\left(\frac{p_{A}^{e}}{p_{v}^{e}}\right)^{i} \exp \left(-\frac{\Gamma_{i, 0}}{k T}\right),
\end{aligned}
$$

which is the proper unary distribution of $A$ clusters in the final equilibrium state over the bulk solution.

Thus, once the equilibrium partitioning between vapor and bulk liquid phases is determined, the cluster distribution can be easily evaluated. The ambiguous normalization constant that arises in the classical derivation is eliminated. Furthermore, the present analysis imposes no assumptions about the nature of the size-dependent correction to the cluster free energy while one might apply the classical capillary approximation assuming that $\Gamma_{i, j}=4 \pi R_{p}^{2} \sigma$, where $\sigma$ is the bulk surface tension, models that fully account for size, shape, compositional, and surface activity effects can readily be integrated into this new formulation. The equilibrium cluster distribution is expressed explicitly in terms of the partial pressures of the vapor species at full thermodynamic equilibrium.

To determine the relevant equilibrium vapor and liquid compositions, consider first a system that initially contains a supersaturated mixture of vapors $A$ and $B$, such that the initial mole fractions of the vapors are $z_{A}$ and $z_{B}$, where $z_{\chi}$ $=p_{\chi} / p$ is the ratio of the partial pressure of gas $\chi$ and $p$ is the total system pressure. (Note that, in the absence of a background gas, the initial pressure would exceed the pressure at equilibrium.) The full thermodynamic equilibrium of this mixture is one in which some of the vapor will have condensed to form a bulk liquid, and some will remain in the vapor phase as monomers and clusters. This is illustrated in the phase diagram for the vapor/liquid system, Fig. 1. In the usual view of the vapor/liquid phase diagram for a mixture, the region above the upper curve would correspond to compressed liquid; here it shows the unstable region of super- saturated vapor from which we seek to establish equilibrium by condensing vapor. The uppermost curve denotes the composition of the liquid that remains when the two phases equilibrate, while the lower curve provides the composition of the vapor. The equilibrium compositions of the liquid and vapor phases will generally differ from that of the initial mixture and from each other, except when the mole fraction of either species equals zero and a single species equilibrium is established. The vapor pressure in the equilibrium state, $p_{v}^{e}$, includes the contributions of $A, B$, and any clusters that may form.

There still remains uncertainty as to what vapor pressure will result from equilibration. It is convenient to express the cluster distribution in terms of the fraction of vapor "molecules" that are clusters of size $\{i, j\}$, which we denote $\phi_{i, j}$. The total vapor concentration is the sum of the contributions of both monomers and clusters of all sizes and compositions, i.e.,

$$
N^{v, e}=N_{A}^{v, e}+N_{B}^{v, e}+N^{v, e} \sum_{k=2}^{\infty} \sum_{i=0}^{k} \phi_{i, k-i},
$$

from which we find

$$
N^{v, e}=\frac{N_{v, A}^{e}+N_{v, B}^{e}}{1-\sum_{k=2}^{\infty} \sum_{i=0}^{k} \phi_{i, k-i}}=\frac{N_{A}^{v, e}+N_{B}^{v, e}}{1-\delta} .
$$

We must now determine the full equilibrium state of the mixed vapor system. To evaluate the equilibrium state of a system that begins with nonequilibrium partial pressures, $p_{A}^{0}$ and $p_{B}^{0}$ and an overall system pressure $p$ maintained by a background gas $C$, we must determine both the compositions and the relative amounts of the vapor and liquid phases. Mole balances on all three species can be expressed as

$$
\begin{aligned}
& N_{A}^{0}=p_{A}^{0} / k T=N_{A}^{l, e}+N_{A}^{v, e}+a\left(N_{A}^{v, e}+N_{B}^{v, e}\right), \\
& N_{B}^{0}=p_{B}^{0} / k T=N_{B}^{l, e}+N_{B}^{v, e}+b\left(N_{A}^{v, e}+N_{B}^{v, e}\right), \\
& N_{C}^{0}=p_{C}^{0} / k T=N_{C}^{v, e} .
\end{aligned}
$$

The contributions of clusters to the mole balances of $A$ and $B$ are denoted by the coefficients $a$ and $b$, respectively, viz.,

$$
\begin{aligned}
& a=\sum_{k=2}^{\infty} \sum_{i=0}^{k} \frac{i \phi_{i, k-i}}{1-\delta}, \\
& b=\sum_{k=2}^{\infty} \sum_{j=0}^{k} \frac{j \phi_{k-j, j}}{1-\delta} .
\end{aligned}
$$

Dividing by the total number of moles in the initial vapor phase, $N^{0}=N_{A}^{0}+N_{B}^{0}+N_{C}^{0}$, and noting that the total numbers of moles of liquid and vapor are $N^{l, e}=N_{A}^{l, e}+N_{B}^{l, e}$ and $N^{v, e}=N_{A}^{v, e}+N_{B}^{v, e}+N_{C}^{v, e}$, respectively, the equilibrium composition can be related to the initial supersaturated vapor via 


$$
\begin{aligned}
& z_{A}=l x_{A}+v\left[(1+a) y_{A}+a y_{B}\right], \\
& z_{B}=l x_{B}+v\left[b y_{A}+(1+b) y_{B}\right], \\
& z_{C}=v y_{c},
\end{aligned}
$$

where $l=N^{l, e} / N^{0}$ and $v=N^{v, e} / N^{0}$, respectively. Summing Eqs. (51)-(53), we find

$$
1=l+v\left[\left(y_{A}+y_{B}\right)(1+a+b)+y_{C}\right] .
$$

The sum of the mole fractions of vapors, clusters, and background gas is

$$
\left(y_{A}+y_{B}\right)(1+\delta)+y_{C}=1,
$$

So

$$
1=l+v\left[1+(a+b-\delta)\left(y_{A}+y_{B}\right)\right] .
$$

The vapor and liquid mole fractions are related through extended Raoult's law, Eq. (38), which may be expressed as

$$
y_{\nu}=\frac{p_{\nu}}{p}=\gamma_{\nu} x_{\nu} \frac{p_{\nu}^{\mathrm{sat}}}{p}=K_{\nu} x_{\nu} .
$$

While a numerical solution to the general problem can be found (see Appendix), Reiss' approximation that clusters contribute little to the mole balance is generally excellent and simplifies the analysis. In the limit of small $a, b$, and $\delta$, Eqs. (51)-(53) can be rearranged to yield

$$
\begin{aligned}
& y_{A} \approx \frac{K_{A} z_{A}}{1-v+v K_{A}}, \\
& y_{B} \approx \frac{K_{B} z_{B}}{1-v+K_{B} v}, \\
& y_{C} \approx \frac{z_{C}}{v} .
\end{aligned}
$$

Noting that the sum of the mole fractions in both the gas and bulk liquid phases must add to unity, the difference between these sums becomes

$$
\frac{z_{A}\left(K_{A}-1\right)}{1-v+v K_{A}}+\frac{z_{B}\left(K_{B}-1\right)}{1-v+v K_{B}}+\frac{z_{C}}{v}=0 .
$$

With minor rearrangement, we obtain a quadratic in $v$, i.e.,

$$
\begin{aligned}
0= & v^{2}\left[\left(z_{A}+z_{B}+z_{C}\right)\left(K_{a}-1\right)\left(K_{B}-1\right)\right]+v\left[\left(z_{A}\left(K_{A}-1\right)\right.\right. \\
& \left.+z_{B}\left(K_{B}-1\right)+z_{C}\left(K_{a}+K_{B}-2\right)\right]+z_{C} .
\end{aligned}
$$

Of the two roots, one satisfies the condition $0 \leqslant v \leqslant 1$, from which the equilibrium state can be fully determined. If $\delta, a$, and $b$ are not negligible, this approximation provides a first approximation from which the true values can be found by iteration using the more general solution presented in the Appendix. Given $v$, the compositions of the vapor and liquid phases at full thermodynamic equilibrium are readily determined.

Returning to the evaluation of the nucleation rate, we now find

$$
\begin{aligned}
F_{i, j}= & \frac{p_{v}^{e}}{k T}\left(\frac{p_{A}^{\mathrm{sat}} \gamma_{A}^{e} x_{A}^{e}}{p_{v}^{e}}\right)^{i}\left(\frac{p_{B}^{\mathrm{sat}} \gamma_{B}^{e} x_{B}^{e}}{p_{v}^{e}}\right)^{j} \\
& \times \exp \left[-\frac{\Gamma_{i, j}}{k T}-i \ln \left(\gamma_{A}^{i, j} x_{A}^{i, j}\right)-j \ln \left(\gamma_{B}^{i, j} x_{B}^{i, j}\right)\right. \\
& \left.+i \ln \frac{\beta_{A}}{\beta_{A}^{e}}+j \ln \frac{\beta_{B}}{\beta_{B}^{e}}\right] \\
= & N^{v, e} \exp \left[i\left\{\ln \left(\frac{p_{A}^{\mathrm{sat}} \gamma_{A}^{e} x_{A}^{e}}{p_{v}^{e}}\right)-\ln \left(\gamma_{A}^{i, j} x_{A}^{i, j}\right)+\ln \frac{\beta_{A}}{\beta_{A}^{e}}\right\}\right. \\
& \left.+j\left\{\ln \left(\frac{p_{B}^{\mathrm{sat}} \gamma_{B}^{e} x_{B}^{e}}{p_{v}^{e}}\right)-\ln \left(\gamma_{B}^{j, j} x_{B}^{i, j}\right)+\ln \frac{\beta_{B}}{\beta_{B}^{e}}\right\}-\frac{\Gamma_{i, j}}{k T}\right] \\
= & N^{v, e} \exp \left[-\frac{\kappa_{i, j}^{e}}{k T}\right] .
\end{aligned}
$$

For ideal vapors, the ratios $\beta_{A} / \beta_{A}^{e}=S_{A}^{e}$ and $\beta_{B} / \beta_{B}^{e}=S_{B}^{e}$ are the saturation ratios of the two vapor species relative to the full thermodynamic equilibrium state rather than relative to the usual pure saturated vapor state. The present thermodynamically consistent approach to the evaluation of the nucleation kinetics replaces the ad hoc specification of the proportionality constant $N_{0}$ with the total vapor concentration, $N^{v, e}$.

The nucleation-rate surface $\kappa_{i, j}$ has a functional form that is similar to that of the reversible work of cluster formation in the constrained equilibrium, i.e., $\omega_{i, j}$, but now the saddle is seen to represent a purely kinetic barrier to stable cluster formation. An additional term is found in the massweighted (i.e., $i, j$ ) portion of the surface, while the surfaceweighted part $\left(\Gamma_{i, j}\right)$ is unchanged. With appropriate thermodynamic data to determine the evaporation rate parameters, the well established methods for calculation of the binary nucleation rate, e.g., Refs. 5-8 and Eq. (16), can be used directly to derive the net nucleation rate from the rate equations derived above.

\section{CONCLUSIONS}

The present paper has shown that the assumption of the constrained-equilibrium cluster distribution in the derivation of the theory of binary or multicomponent nucleation is an unnecessary artifice that can be eliminated by applying the law of mass action at a state of full thermodynamic equilibrium of the starting mixture. The specification of a rational equilibrium state further eliminates ambiguity in the scaling factor (reference concentration), and produces consistent results in the limit of single vapor nucleation. The mathematical form of the cluster rate equations in this new derivation is similar to that produced by the classical binary nucleation theory, so the calculation of the nucleation rate will follow the same form as in the traditional treatment. The simplifications introduced through the capillarity approximation can be introduced, but extension to models that more accurately account for the variation of the cluster free energy with cluster size and surface activity effects on the cluster free energy is also possible. 


\section{ACKNOWLEDGMENTS}

The author gratefully acknowledges insightful discussions with Professor John Seinfeld, Professor Zhen-Gang Wang, and Professor George Gavalas of the California Institute of Technology, and Professor Joseph Katz of the Johns
Hopkins University. This work was supported in part by the National Science Foundation Grant No. ATM-0340832.

\section{APPENDIX: GENERAL SOLUTION}

Solving for the individual contributions to the vapor phase yields

$$
\begin{aligned}
& y_{A}=\frac{K_{A} K_{B} v a z_{B}-K_{A} z_{A}\left[1-(1+W) v+K_{B} v(1+b)\right]}{K_{A} K_{B} v^{2} a b-\left[1-(1+W) v+K_{A} v(1+a)\right]\left[1-(1+W) v+K_{B} v(1+b)\right]}, \\
& y_{B}=\frac{K_{A} K_{B} v b z_{A}-K_{B} z_{B}\left[1-(1+W) v+K_{A} v(1+a)\right]}{K_{A} K_{B} v^{2} a b-\left[1-(1+W) v+K_{A} v(1+a)\right]\left[1-(1+W) v+K_{B} v(1+b)\right]}, \\
& y_{C}=\frac{z_{C}}{v},
\end{aligned}
$$

where $W=a+b-\delta$. Each of the species mole fractions can now be determined from the appropriate equilibrium constants, and the number of moles of vapor per mole of initial mixture, subject to the constraint that the sums of the mole fractions in each phase must be unity. To solve for $v$, we let the difference between the sums of mole fractions be zero, i.e.,

$$
0=y_{A}+y_{B}+y_{C}-x_{A}-x_{B}=y_{A}\left(1-K_{A}^{-1}\right)+y_{B}\left(1-K_{B}^{-1}\right)+y_{C},
$$

or

$$
\begin{aligned}
0= & Z_{A}\left(K_{A}-1\right)+Z_{B}\left(K_{B}-1\right)+z_{C}-v\left[z_{A}\left(K_{A}-1\right)\left(K_{B}(1+b)-(1+W)\right)+Z_{B}\left(K_{B}-1\right)\left(K_{A}(1+a)-(1+W)\right)\right. \\
& \left.+z_{C}\left(2(1+W)-K_{A}(1+a)-K_{B}(1+b)\right)\right]+v^{2}\left\{z_{A}\left[b K_{A}\left(K_{B}-1\right)\right]+z_{B}\left[a K_{B}\left(K_{A}-1\right)\right]+z_{C}(1+W)\right. \\
& \left.\times\left[K_{A}(1+a)+K_{B}(1+b)-K_{A} K_{B}(1+a+b)-(1+W)\right]\right\},
\end{aligned}
$$

which is quadratic in $v$ and can be solved directly. Iteration is required to obtain the proper values for $a, b, \delta$, and $W$.

${ }^{1}$ H. Vehkamäki, M. Kulmala, I. Napari, K. Lehtinen, C. Timmreck, M. Noppel, and A. Laaksonen, J. Geophys. Res., [Atmos.] 107, 4622 (2002).

${ }^{2}$ M. Boy and M. Kulmala, Atmos. Chem. Phys. 2, 1 (2002).

${ }^{3}$ M. Kulmala, H. Vehkamäki, T. Petajda, M. Dal Maso, A. Lauri, V. Kerminen, W. Birmili, and P. McMurry, J. Aerosol Sci. 35, 143 (2004).

${ }^{4}$ Q. Zhang, C. Stanier, M. Canagaratna, J. Jayne, D. Worsnop, S. Pandis, and J. Jimenez, Environ. Sci. Technol. 38, 4797 (2004).

${ }^{5}$ H. Reiss, J. Chem. Phys. 18, 840 (1950).

${ }^{6}$ D. Stauffer, J. Aerosol Sci. 7, 319 (1976).

${ }^{7}$ H. Trinkaus, Phys. Rev. B 27, 7372 (1983).

${ }^{8}$ G. Wilemski, J. Chem. Phys. 110, 6451 (1999).

${ }^{9}$ A. Laaksonen, M. Kulmala, and P. Wagner, J. Chem. Phys. 99, 6832 (1993).
${ }^{10}$ G. Wilemski and B. Wyslouzil, J. Chem. Phys. 103, 1127 (1995).

${ }^{11}$ R. McGraw, J. Chem. Phys. 102, 2098 (1995).

${ }^{12}$ B. Hale and G. Wilemski, Chem. Phys. Lett. 305, 263 (1999).

${ }^{13}$ M. Kulmala, A. Laaksonen, and S. L. Girshick, J. Aerosol Sci. 23, 309 (1992).

${ }^{14}$ A. Laaksonen and D. W. Oxtoby, J. Chem. Phys. 102, 5803 (1995).

${ }^{15}$ I. Kusaka and D. W. Oxtoby, J. Chem. Phys. 115, 4883 (2001).

${ }^{16}$ Y. Viisanen, R. Strey, A. Laaksonen, and M. Kulmala, J. Chem. Phys. 100, 6062 (1994)

${ }^{17}$ S. P. Fisenko and G. Wilemski, Phys. Rev. E 70, 056119 (2004).

${ }^{18}$ J. L. Katz and H. Wiedersich, J. Colloid Interface Sci. 61, 351 (1977).

${ }^{19}$ J. L. Katz and F. Spaepen, Philos. Mag. B 37, 137 (1978).

${ }^{20}$ J. Katz and M. Donohue, J. Colloid Interface Sci. 85, 267 (1982).

${ }^{21}$ A. Laaksonen, R. McGraw, and H. Vehkamäki, J. Chem. Phys. 111, 2019 (1999).

${ }^{22}$ J. E. McDonald, Am. J. Phys. 30, 870 (1962). 